\title{
Revisores consultados (dezembro/2020 a novembro/2021)
}

Adela Gueller, Pontifícia Universidade Católica de São Paulo, SP, Brasil Adriana Barbosa Pereira, Pontifícia Universidade Católica de São Paulo, SP, Brasil Adriana Leão, Universidade Federal do Espírito Santo, ES, Brasil Alessandra Sapoznik, Complutense University of Madrid, Espanha Alessandro de Oliveira dos Santos, Universidade de São Paulo, SP, Brasil Alexandre Jordão, Sociedade de Psicanálise da Cidade do Rio de Janeiro, RJ, Brasil Amadeu Weinmann, Universidade Federal do Rio Grande do Sul, RS, Brasil Amilton Santos Jr., Universidade Estadual de Campinas, SP, Brasil Ana Lucia Carvalho, Universidade Federal da Paraiba, PB, Brasil Ana Karina Fachini Araújo, Instituto Sedes Sapientiae, SP, Brasil Ana Maria Jacó-Vilela, Universidade Estadual do Rio de Janeiro, RJ, Brasil Ana Maria Raimundo Oda, Universidade Estadual de Campinas, SP, Brasil Ana Paula Lacorte Gianesi, Fórum do Campo Lacaniano de São Paulo, SP, Brasil Analice Palombini, Universidade Federal do Rio Grande do Sul, RS, Brasil André Ehrlich, Universidade Tuiuti do Paraná, PR, Brasil Andrea Ferrari, Universidade Federal do Rio Grande do Sul, RS, Brasil Andrea Gallassi, Universidade de Brasília, DF, Brasil Beatriz Santos, Université Paris, França Benilton Bezerra, Universidade Estadual do Rio de Janeiro, RJ, Brasil Bruna Brito, Universidade Federal Fluminense, RJ, Brasil Carlos Alberto Pinto, Pontifícia Universidade Católica de Minas Gerais, MG, Brasil Clarissa de Rosalmeida Dantas, Universidade Estadual de Campinas, SP, Brasil Claudia Andrade, Universidade Federal do Estado do Rio de Janeiro, RJ, Brasil Claudio Banzato, Universidade Estadual de Campinas, SP, Brasil

Cristina Nogueira, Associação Mundial de Psicanálise, Brasil Cynara Ribeiro, Universidade Federal do Rio Grande do Norte, RN, Brasil 
Daniel Camparo Avila, Universidad de la República Uruguay, Uruguai Daniel Delouya, Sociedade Brasileira de Psicanálise de São Paulo, SP, Brasil Daniela Scheinkman Chatelard, Universidade de Brasília, DF, Brasil Daniela Schneider, Universidade Federal de Santa Catarina, SC, Brasil Debora Uhr, Universidade Federal do Rio de Janeiro, RJ, Brasil

Deborah Lima Klajnman, Universidade do Estado do Rio de Janeiro, RJ, Brasil Deise Amparo, Universidade de Brasília, DF, Brasil Elisabeth Juliboni, Universidade Federal Fluminense, RJ, Brasil Elizabeth Robin Zenkner Brose, Universidade de São Paulo, SP, Brasil Elizabeth Cristina Landi, Pontifícia Universidade Católica de Goiás, GO, Brasil Erico Bruno Viana Campos, Universidade Estadual de São Paulo, SP, Brasil Erotildes Maria Leal, Universidade Federal do Rio de Janeiro, RJ, Brasil Eugênio Canesin Dal Molin, Centro Universitário Filadélfia, PR, Brasil Everton Fernandes, Faculdade de Ciências Médicas de Minas Gerais, MG, Brasil Fátima Caropreso, Universidade Federal de Juiz de Fora, MG, Brasil Fatima Regina Machado, Universidade de São Paulo, SP, Brasil Fernanda Freire Pimentel, Universidade Estadual do Rio de Janeiro, RJ, Brasil Fernando M. F. Silva, Universidade de Lisboa, Portugal

Flávio Durães, Pontifícia Universidade Católica de Minas Gerais, MG, Brasil Fuad Kyrillos Neto, Universidade Federal de São João Del-Rei, MG, Brasil Gabriela Xavier de Araújo, Universidade Federal do Rio Grande do Sul, RS, Brasil Gariella Dupim, Universidade Federal de Campina Grande, PB, Brasil Glaucia Ribeiro Starling Diniz, Universidade de Brasília, DF, Brasil Guilherme Geha dos Santos, Universidade Estadual de Maringá, PR, Brasil Gustavo Bonini Castellana, Universidade de São Paulo, SP, Brasil Gustavo H. Dionísio, Universidade Estadual Paulista, SP, Brasil Hélio Miranda, Pontifícia Universidade Católica de Minas Gerais, MG, Brasil Iara Richwin Ferreira, Universidade de Brasília, DF, Brasil Ivan Ramos Estevão, Universidade de São Paulo, SP, Brasil Jackeline Romio, Universidade de São Paulo, SP, Brasil Jairo Gama, Universidade Federal de Juiz de Fora, MG, Brasil Jaqueline da Silva, Universidade Federal do Rio de Janeiro, RJ, Brasil Jean Marcos Pinheiro Borba, Universidade Federal do Maranhão, MA, Brasil João Leite Ferreira Neto, Pontifícia Universidade Católica de Minas Gerais, MG, Brasil Júlia Catani, Universidade de São Paulo, SP, Brasil Juliana Farah, Universidade de São Paulo, SP, Brasil Karen Scavacini, Instituto Vita Alere de Prevenção e Posvenção do Suicídio, SP, Brasil 


\section{REVISORES CONSULTADOS (2020/2021)}

Karla Patrícia Holanda Martins, Universidade Federal do Ceará, CE, Brasil

Laurinda Rosa Maciel, Casa de Oswaldo Cruz, RJ,Brasil

Leonar Goldberg, Universidade Brasil, Brasil

Leonardo de Miranda Ferreira, Universidade do Estado do Rio de Janeiro, RJ, Brasil

Leopoldo Fulgencio, Universidade de São Paulo, SP, Brasil

Lilian Miranda, Fundação Oswaldo Cruz, RJ, Brasil

Lucas Bulamah, Universidade de São Paulo, SP, Brasil

Lucia Maria de Freitas Perez, Universidade Federal do Estado do Rio de Janeiro, RJ, Brasil

Lucia Rosa, Universidade Federal do Piauí, PI, Brasil

Luis Achilles, Universidade Federal do Paraná, PR, Brasil

Luis Guilherme Streb, Hospital Conceição, RS, Brasil

Luis Sanfelippo, Universidad Nacional de la Plata, Argentina

Luiz Carlos Tarelho, Fondation Jean Laplanche, França

Luiz Fernando Farah de Tofolli, Universidade Estadual de Campinas, SP, Brasil

Macla Ribeiro Nunes, Universidade do Estado do Rio de Janeiro, RJ, Brasil

Mara Caffé, Instituto Sedes Sapientiae, SP, Brasil

Marcelo Duarte Porto, Universidade Estadual de Goiás, GO, Brasil

Marcia Aparecida Ferreira de Oliveira, Universidade de São Paulo, SP, Brasil

Maria da Graça M Gonçalves, Pontifícia Universidade Católica de São Paulo, SP, Brasil

Maria Inês Fernandes, Universidade de São Paulo, SP, Brasil

Mariana L. Afonso, Universidade de São Paulo, SP, Brasil

Marilia Etienne Arreguy, Universidade Federal Fluminense, RJ, Brasil

Mario Eduardo Pereira, Universidade Estadual de Campinas, SP, Brasil

Marta Regina de LeãoD'Agord, Universidade Federal do Rio Grande do Sul, RS, Brasil Maurício Viotti Daker, Universidade Federal de Minas Gerais, MG, Brasil

Mélinda Marx, Université de Rennes 2 UFR Sciences Sociales, França

Michel Haddad, Universidade Federal de São Paulo, SP, Brasil

Nuria Malajovich Muñoz, Universidade Federal do Rio de Janeiro, RJ, Brasil

Octavio Domont de Serpa Junior, Universidade Federal do Rio de Janeiro, RJ, Brasil

Paula Brant, Prefeitura Municipal de Belo Horizonte, MG, Brasil

Paulo Roberto Ceccarelli, Pontifícia Universidade Católica de Minas Gerais, MG, Brasil Paulo Sérgio de Souza Jr, Universidade Estadual de Campinas, SP, Brasil

Pedro Ambra, Pontifícia Universidade Católica de São Paulo; Universidade de São Paulo, SP, Brasil

Pedro Efken, Universidade Católica de Pernambuco, PE, Brasil 
Pedro Gabriel Delgado, Universidade Federal do Rio de Janeiro, RJ, Brasil

Pedro Muñoz, Pontifícia Universidade Católica do Rio de Janeiro, RJ, Brasil

Rafael Alves Lima, Universidade de Sao Paulo, SP, Brasil

Raudelio Machin Suárez, Universidad Andres Bello, Chile

Renata Riguini, Pontifícia Universidade Católica de Minas Gerais, MG, Brasil

Renato Palma, Universidade do Estado do Rio de Janeiro, RJ, Brasil

Renato Diniz Silveira, Pontifícia Universidade Católica de Minas Gerais, MG, Brasil

Ricardo Maia Júnior, CLIO - Associação de Psicanálise, CE, Brasil

Richard Simanke, Universidade Federal de Juiz de Fora, MG, Brasil

Rinaldo Voltolini, Universidade de São Paulo, SP, Brasil

Rita Manso, Universidade Federal do Estado do Rio de Janeiro, RJ, Brasil

Roberto Tykanori, Universidade Federal de São Paulo, SP, Brasil

Rodrigo Alencar, Instituto Vox de Pesquisa em Psicanálise, SP, Brasil

Rodrigo Gonsalves, The European Graduate School, Suíça

Rosa Guedes Lopes, Universidade Veiga de Almeida, RJ, Brasil

Rosana Coelho, Universidade do Estado do Rio de Janeiro, RJ, Brasil

Rose Gurski, Universidade Federal do Rio Grande do Sul, RS, Brasil

Sabrina Stefanello, Universidade Federal do Paraná, PR, Brasil

Solange Aparecida Nappo, Universidade Federal de São Paulo, SP, Brasil

Solange Fuchs, Grupo Brasileiro de Pesquisas Sandor Ferenczi, RJ, Brasil

Sonia Leite, Universidade do Estado do Rio de Janeiro, RJ, Brasil

Sonia Xavier de Almeida Borges, Universidade Veiga de Almeida, RJ, Brasil

Stephan Malta Oliveira, Universidade Federal do Rio de Janeiro, RJ, Brasil

Susane Zanotti, Universidade Federal de Alagoas, AL, Brasil

Suzana Barroso, Pontifícia Universidade Católica de Minas Gerais, MG, Brasil

Tales Ab'Saber, Universidade Federal de São Paulo, SP, Brasil

Tania Grigolo, CESUSC College, SC, Brasil

Victor Alexandre Garcia Pires, Pontifícia Universidade Católica do Rio de Janeiro, RJ, Brasil

Vinicius Darriba, Universidade Estadual do Rio de Janeiro, RJ, Brasil

Wallace Xavier, Prefeitura Municipal de Belo Horizonte, MG, Brasil

Wellington Zangari, Universidade de São Paulo, SP, Brasil

Wilson Franco, Laboratório PsiA, SP, Brasil 